\title{
Self-association dynamics and morphology of short polymeric PBA-b/co-PAA surfactants
}

\section{Journal Article}

\section{Author(s):}

Lamprou, Alexandros; Xie, Delong; Storti, Giuseppe (i); Wu, Hua (1)

Publication date:

2014-03

Permanent link:

https://doi.org/10.3929/ethz-b-000081810

Rights / license:

In Copyright - Non-Commercial Use Permitted

Originally published in:

Colloid \& polymer science 292(3), https://doi.org/10.1007/s00396-013-3119-2 


\title{
Self-association dynamics and morphology of short polymeric PBA-b/co-PAA surfactants
}

\author{
Alexandros Lamprou • Delong Xie • Giuseppe Storti • \\ Hua Wu
}

Received: 20 September 2013 / Accepted: 5 November 2013 /Published online: 21 November 2013

(C) Springer-Verlag Berlin Heidelberg 2013

\begin{abstract}
The self-association characteristics of very short and well-defined poly(butyl acrylate)-b-poly(acrylic acid) (PBA-b-PAA) block copolymers in water have been studied. The diblocks are asymmetric with the PBA block longer than the PAA block, giving rise to hollow sphere morphology. This is affirmed by experimental data and theoretical evaluations of the hydrophilic and hydrophobic domain sizes, as well as a value close to 1 for the ratio of the hydrodynamic to the gyration radius of the micelles. Besides, the untypically short PBA blocks (polymerization number around 15) render the micelles dynamic. Indications in support include among others the following: the CMC (critical micellar concentration) values depend, together with the aggregation numbers and the micellar sizes, on the block lengths, as predicted by theory; above the CMC their sizes are concentrationindependent, while the micelles disappear below CMC. A comparison was also made with a random PBA-co-PAA copolymer of similar length, which self-associates at an apparent CMC 1 order of magnitude larger than those of the block copolymers, but the size of the formed micelles depends on the concentration.
\end{abstract}

Keywords Self-assembly dynamics $\cdot$ Micelle morphology · Vesicle $\cdot$ Block copolymer $\cdot$ PBA-b-PAA $\cdot$ Polymeric surfactants $\cdot$ ATRP

Electronic supplementary material The online version of this article (doi:10.1007/s00396-013-3119-2) contains supplementary material, which is available to authorized users.

A. Lamprou $\cdot$ D. Xie $\cdot$ G. Storti $\cdot$ H. Wu $(\bowtie)$

Department of Chemistry and Applied Biosciences, ETH Zurich, 8093 Zurich, Switzerland

e-mail: hua.wu@chem.ethz.ch

\section{Introduction}

Well-defined amphiphilic copolymers exhibit a wide range of potential applications, including colloidal stabilization and destabilization, friction reduction, viscosity and wetting modification, as well as applications in pharmaceutics and biomedicines like drug delivery and gene therapy [1-3]. The hydrophobicity, glass transition temperature, charges, number and distribution of the constituting monomer units along the copolymer chain (block/gradient/ random) are all crucial for the self-organization and surface activity of the copolymer, thus affecting their application behavior. While the properties of a random copolymer are expected to be an average of the properties of its constituting units, those of a block-copolymer are substantially different [4]. For diblock copolymers, a variety of self-assembled structures has been observed, with spherical micelles being the most typical [2]. When the block copolymer is asymmetric in favor of its solvophobic block, the so-called "crew-cut micelles" are obtained, with one of the possible morphologies, hollow spheres (vesicles) [2, 5-10].

Recently, an issue receiving particular attention in the literature is dynamics of block copolymer self-assemblies in aqueous solutions, which can be critical for applications such as drug release and latex stabilization $[3,9,11]$. Contrary to the initial expectations coming from the behavior of conventional surfactants, the aggregates formed by diblock copolymers are often "frozen", i.e., out of equilibrium structures. This was proven to be the case not only for block copolymers with glassy hydrophobic blocks, like polystyrene (PS) (physically frozen) [11, 12], but also for those with rubbery blocks, like poly ( $n$-butyl acrylate) (PBA) (kinetically frozen) [11, 13-19]. Whether a system is dynamic or frozen is essentially governed by the height of the energy barrier $\left(E_{\mathrm{a}}\right)$ for extracting a unimer from the core of a micelle, which scales with [20] $E_{\mathrm{a}} \propto n^{2 / 3} \gamma$, where $n$ is the polymerization number of the solvophobic block and $\gamma$ is its interfacial tension with the 
solvent [15-17]. Strategies that have been pursued to reduce $E_{\text {a }}$ are incorporation of hydrophilic units (e.g., acrylic acid (AA) $[12,18,19,21])$ in the hydrophobic block, or replacement with a less hydrophobic [16, 17] or thermosensitive [22] polymer.

In the case of poly(butyl acrylate)-b-poly(acrylic acid) (PBA-b-PAA) block copolymers, comprehensive discussion can be found in the literature about their micellization dynamics and surface activity in aqueous solutions [13-19, 21]. The general conclusion is that the micelles formed by PBA-b-PAA in aqueous solution are kinetically frozen. For example, Jacquin et al. [15] systematically investigated the properties of various PBA-b-PAA block copolymers with the polymerization number of the PBA and PAA blocks in the range of 20-55 and 50-500, respectively, and concluded that in all the cases, their aqueous dispersions are kinetically frozen. Nevertheless, since in principle a shorter hydrophobic PBA block would favor the mobility of the unimers within the aggregates [16], a question arises naturally whether the aggregates become dynamic if we further shorten the PBA block to have the polymerization number of the PBA block smaller than 20. It should be mentioned that such a question is also of direct practical relevance. Recently, we have applied short PBA-bPAA copolymers as surfactants to stabilize polymer particles [3], and found their superior performance with respect to conventional ionic surfactants. Thus, further insight into their self-assembly behavior would help us to better control their performance.

Answering the above question becomes one of the objectives in this work. To this aim, we have prepared well-defined PBA-b-PAA block copolymers with the polymerization number of the hydrophobic PBA block being around 15. Moreover, the hydrophilic PAA blocks were designed even shorter, less than 10 AA units, leading to asymmetric diblock copolymers. It will be seen that with these asymmetric diblock copolymers, the micelles exhibit the morphology of hollow, instead of homogeneous, spheres. In addition, a random PBAco-PAA copolymer of a similar size has been designed as well, and the properties of its aqueous solutions have been also studied and compared with those of the diblock copolymers.

\section{Experimental}

\section{The PBA-b/co-PAA surfactants}

To synthesize well-defined polymeric PBA-b/co-PAA surfactants, we applied the Atom Transfer Radical Polymerization (ATRP) technique, based on a procedure reported by Colombani et al. [13], but modified in order to yield the desired molecular weights and monomer sequences (block or random copolymers). In short, ATRP of $n$-butyl acrylate for the case of a block-copolymer, together with $t$-butyl acrylate (tBA) for a random copolymer, was initiated by methyl $\alpha$-chloropropionate employing $\mathrm{Cu}(\mathrm{I}) \mathrm{Br}$ and $1,1,4,7$, 10,10-hexa-methyl-triethylene-tetramine (HMTETA) as catalyst in $25 \%$ ethyl acetate solution at $70^{\circ} \mathrm{C}$, or in acetone at $60^{\circ} \mathrm{C}$ for a random copolymer. The obtained PnBA chains were reinitiated in $25 \%$ acetone solution at $60^{\circ} \mathrm{C}$ to grow a PtBA block. The resulting block or random copolymers (PnBA-b/co-PtBA) were treated with trifluoroacetic acid (TFA) in dichloromethane in order to deprotect the $t$-butyl groups towards AA units. After each ATRP step the respective intermediate product was purified by flash chromatography through a silica column and was characterized by ${ }^{1} \mathrm{H}$ NMR, GPC and elemental analysis. The final products were characterized by ${ }^{1} \mathrm{H}$ NMR, while the residual copper amount was determined by atomic absorption spectroscopy (AAS).

We have attempted to keep the number of BA units approximately the same, around 15 , and to vary the number of AA units so as to achieve AA molar fractions between $25 \%$ and $50 \%$. In reality, we obtained two asymmetric PBA-bPAA block copolymers with the numbers of BA and AA units equal to 15 and 5 , and 13 and 9 , referred to as $B_{15-5}$ and $B_{13-9}$, respectively. Thus, the AA molar fractions are $25 \%$ and $41 \%$, respectively, for $\mathrm{B}_{15-5}$ and $\mathrm{B}_{13-9}$, which are significantly asymmetric. The obtained random PBA-co-PAA copolymer is composed of $13 \mathrm{BA}$ and $14 \mathrm{AA}$ units, referred to as $\mathrm{R}_{13-14}$. It should be noted that the PBA-co-PAA copolymer is a statistical copolymer, owing to the same reactivity of $n$-BA and $t$-BA [18]. The molecular characteristics of the synthesized copolymers are summarized in Table 1, while more details regarding the synthesis and molecular characterization are available in the Supplementary material (SM).

The copolymers were dissolved in doubly deionized water with the solution $\mathrm{pH}$ adjusted to 7 by $\mathrm{NaOH}$, followed by filtration through a 200-nm PVDF filter. The obtained surfactant stock solutions were used to prepare solutions or dispersions for subsequent measurements.

\section{CMC of the surfactants}

The CMC (critical micellar concentration) of the synthesized polymeric surfactants was determined via the regular surface tension method, where the surface tension of the surfactant solution was measured as a function of the surfactant concentrations with a Wilhelmy plate tensiometer (DCAT21, dataphysics). All the solutions were prepared at least $24 \mathrm{~h}$ before the measurements.

\section{Static and dynamic light scattering}

The static light scattering (SLS), based on the Zimm plot, was applied to evaluate the size and aggregation number of micelles. To this aim, the required refractive index increment $\mathrm{d} n$ / $\mathrm{d} C$ of each copolymer was determined via a chromatographic 
Table 1 Molecular characteristics of synthesized PBA-b/co-PAA surfactants

\begin{tabular}{llllllll}
\hline Surfactant & $x_{\mathrm{AA}}$ & $n_{\mathrm{t}}$ & $M_{\mathrm{n}}\left(\mathrm{g} \mathrm{mol}^{-1}\right)$ & $\mathrm{PDI}$ & $\mathrm{CMC}\left(\mathrm{mol} \mathrm{l}^{-1}\right)$ & $\mathrm{CMC}(\mathrm{wt} . \%)$ & $\mathrm{d} n / \mathrm{d} C\left(\mathrm{mg} \mathrm{ml} l^{-1}\right)$ \\
\hline $\mathrm{B}_{15-5}$ & 0.25 & 20 & 2,405 & 1.31 & $9.10 \times 10^{-5}$ & $2.19 \times 10^{-2}$ & 0.104 \\
$\mathrm{~B}_{13-9}$ & 0.41 & 22 & 2,437 & 1.35 & $1.08 \times 10^{-4}$ & $2.64 \times 10^{-2}$ & 0.112 \\
$\mathrm{R}_{13-14}$ & 0.52 & 27 & 2,798 & 1.22 & $7.75 \times 10^{-4}$ & $2.17 \times 10^{-1}$ & 0.079 \\
\hline
\end{tabular}

$n_{t}$ total polymerization number of $\mathrm{BA}+\mathrm{AA}, x_{A A}$ molar fraction of acrylic acid in copolymer, $M_{\mathrm{n}}$ number average molecular weight by NMR, $P D I$ polydispersity index by GPC

RI method [23]. The RI signal of polymer solutions at various concentrations, $C$, was monitored using a Hitachi L-7000 HPLC system, equipped with autosampler, isocratic pump and $\mathrm{RI}$ detector, operated at $25^{\circ} \mathrm{C}$ with $\mathrm{H}_{2} \mathrm{O}$ as mobile phase. For each polymer solution a blank solution of identical solvent composition was used as reference, while $\mathrm{NaCl}$ solutions were used for calibrating the detector. The obtained $\mathrm{d} n / \mathrm{d} C$ values for the three copolymers are listed in Table 1.

All light scattering measurements were performed at $25^{\circ} \mathrm{C}$ in cylindrical quartz cuvettes, illuminated by a $\lambda=532 \mathrm{~nm}$ laser beam emitted from a solid-state laser (Ventus LP532 Laser Quantum), while the detection angle $\theta$ was controlled by a BI-200SM goniometer (Brookhaven Instruments). The corresponding magnitude of the scattering vector is defined as $q=$ $4 \pi n / \lambda \sin (\theta / 2)$, where $n$ is the refractive index of the medium.

SLS was performed at scattering angles ranging from $20^{\circ}$ to $150^{\circ}$. Each measurement range was usually repeated three times. From the reduced Raleigh expression, the radius of gyration $R_{\mathrm{g}}$ and the weight-average molecular mass, $M_{\mathrm{w}}$, of the scattering micelles have been determined through the following Zimm plot, where the intercept of the straight line when both $C \rightarrow 0$ and $\theta \rightarrow 0$ yields $M_{\mathrm{w}}$ and the slope when $C \rightarrow 0$ yields $R_{\mathrm{g}}$ :

$\frac{K C}{R_{\theta}}=\frac{1}{M_{\mathrm{w}}}\left(1+\frac{1}{3} q^{2} R_{\mathrm{g}}^{2}\right)+2 A_{2} C$,

where $A_{2}$ is the second Virial coefficient, $R_{\theta}$ is the excess Rayleigh ratio, which is expressed using that of toluene:

$R_{\theta}=\frac{I-I_{\mathrm{sol}}}{I_{\mathrm{T}}}\left(\frac{n}{n_{\mathrm{T}}}\right)^{2} R_{\mathrm{T}}$,

where $I, I_{\mathrm{sol}}$ and $I_{\mathrm{T}}$ are the scattered intensities of the sample, solvent and toluene, respectively, while $n_{\mathrm{T}}$ and $R_{\mathrm{T}}$ are the refractive index and Rayleigh ratio of toluene, equal to 1.496 and $2.1 \times 10^{-5} \mathrm{~cm}^{-1}$, respectively [24]. The quantity, $K$ in Eq. 1, is an optical constant defined as:

$K=\frac{2 \pi^{2}}{\lambda^{4} N_{\mathrm{A}}}\left(n \frac{\mathrm{d} n}{\mathrm{~d} C}\right)^{2}$,

where $N_{\mathrm{A}}$ is the Avogadro number.
For further elucidation of the micelle size, polarized (VV) dynamic light scattering (DLS) was performed at $90^{\circ}$. The possibility of anisotropy was examined by depolarized (VH) DLS at scattering angles between $60^{\circ}$ and $145^{\circ}$. The intensity autocorrelation functions $C(\tau)$ were generated by a BI9000AT digital correlator and processed by the corresponding Brookhaven Instruments software. Thus, by applying the typical cumulant fitting on the obtained distribution of decay rates, $\Gamma$, one can determine the average hydrodynamic radius, $R_{\mathrm{h}}$.

Cryo-TEM imaging was performed following an experimental protocol described elsewhere [25].

\section{Results and discussion}

Surface activity and CMC

All synthesized amphiphilic copolymers are readily dispersible in water at $\mathrm{pH} 7.0$ and surface-active, inducing a reduction in the surface tension of the air-water interface, as shown in Fig. 1. The change in the slope of the surface tension curve in Fig. 1 occurs when the copolymer concentration reaches a certain value, which corresponds to the $\mathrm{CMC}$ and indicates the formation of self-assembled structures. The obtained $\mathrm{CMC}$ values are listed in Table 1. It is seen that when the PBA length decreases from 15 to 13 and the PAA length increases from 5 to 9 , the $\mathrm{CMC}_{\text {of }} \mathrm{B}_{13-9}$ increases by $20 \%$ with respect to that of $\mathrm{B}_{15-5}$. In fact, it is well established in the literature [8, 15, $17,26,27]$ that both shortening the solvophobic block and increasing the solvophilic block increase the CMC, if the solvophobic block is very short, i.e., below 30 units, a trend attributed to increasing solubility.

Let us now compare the measured CMC values of our block copolymers with the theoretical estimate from the literature. It is known that the CMC value of a block copolymer can be approximated by the solubility limit of its solvophobic block, provided that the interfacial tension $\gamma$ between the solvophobic block and the solvent is high enough, so that the contribution of its solvophilic corona can be neglected. This is valid for PBA-b-PAA in water, for which $\gamma_{\mathrm{PBA} / \mathrm{H}_{2} \mathrm{O}}=2.0 \times 10^{-6} \mathrm{~J} \mathrm{~cm}^{-2}$ and the $\mathrm{CMC}$ can be approximated by [15] 

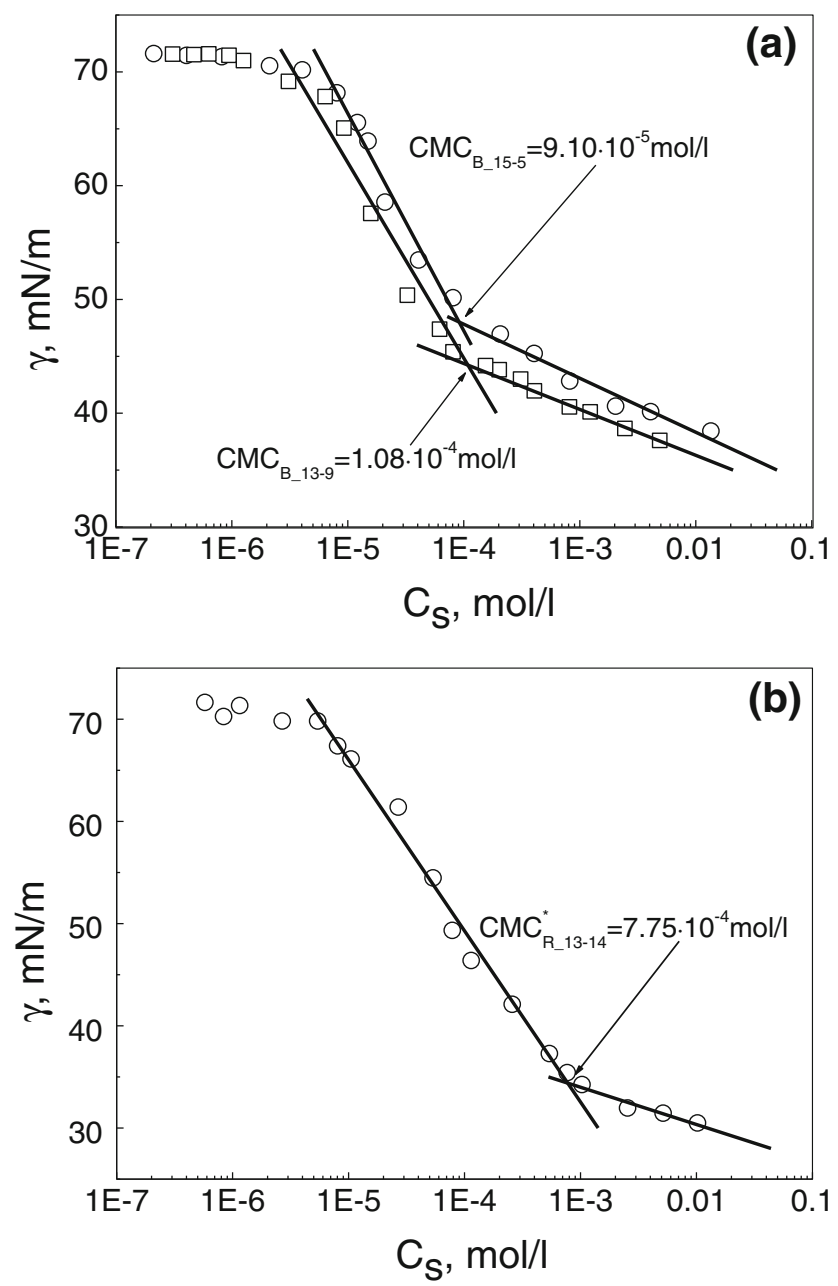

Fig. 1 Semi-logarithmic plots of the measured surface tension as a function of the surfactant concentration for a $\mathrm{B}_{15-5}$ (open circle) and $\mathrm{B}_{13-9}$ (empty square) and $\mathbf{b} \mathrm{R}_{13-14}$ at $T=25^{\circ} \mathrm{C}$

$\mathrm{CMC} \approx \frac{100 M_{\mathrm{BA}} x_{\mathrm{BA} / \mathrm{AA}}}{v_{0} N_{\mathrm{A}}\left(1+x_{\mathrm{BA} / \mathrm{AA}}\right)} \exp \left[-\frac{E_{\mathrm{a}}}{k_{\mathrm{B}} T}\right]$

with

$E_{\mathrm{a}}=\pi^{1 / 3}\left(\frac{3}{4} n_{\mathrm{PBA}} v_{0}\right)^{2 / 3} \cdot \gamma_{\mathrm{PBA} / \mathrm{H}_{2} \mathrm{O}}$

where $M_{\mathrm{BA}}\left(\mathrm{g} \mathrm{mol}^{-1}\right)$ is the molar mass of the PBA block, $x_{\mathrm{BA} /}$ $\mathrm{AA}$ is the mass ratio of $\mathrm{BA}$ over $\mathrm{AA}$ in the copolymer, $v_{0}$ is the molecular volume of a single BA unit, which can be estimated from PBA density at $T=298 \mathrm{~K}\left(\rho_{\mathrm{PBA}}=1.10 \mathrm{~g} \mathrm{~cm}^{-3}\right.$ at $T=25^{\circ} \mathrm{C}$ [28]), thus with a value of $v_{0}=1.93 \times 10^{-22} \mathrm{~cm}^{3}$. With the polymerization number of the PBA block, $n_{\mathrm{PBA}}$ (15 and 13) for $\mathrm{B}_{15-5}$ and $\mathrm{B}_{13-9}$, the computed $\mathrm{CMC}$ values are $3.74 \times 10^{-5}$ and $8.08 \times 10^{-5} \mathrm{~mol} / 1$, respectively. These values are very comparable to the measured ones in Table 1 , not only in the orders of magnitude and trend, but - in the case of $\mathrm{B}_{13-9}$ - also in the absolute value, thus supporting the reliability of the measured $\mathrm{CMC}$ values.

The random copolymer, $R_{13-14}$, is essentially a polyelectrolyte. Such copolymers often form aggregates in water [4], exhibiting an "apparent" CMC [12]. These are for instance the cases for the solutions of poly(styrene)-co-PAA,[29, 30] poly(dodecyl acrylate)-co-PAA alkali-soluble resins (ASR) [31], and $\mathrm{P}(\mathrm{S}-c o-\mathrm{AA})-\mathrm{b}-\mathrm{PAA}$ copolymers with $50 \% \mathrm{AA}$ in the hydrophobic block [12]. This behavior can be attributed to the inter- and intra-molecular association of the hydrophobic units in the presence of water [32]. For our $\mathrm{R}_{13-14}$, the CMC value is $7.75 \times 10^{-4} \mathrm{~mol} / \mathrm{l}$, which is well in the range of the $\mathrm{CMC}$ values reported for PS-co-PAA ASR [30]. The CMC value of $\mathrm{R}_{13-14}$ is almost one order of magnitude larger than those of the two block copolymers, $\mathrm{B}_{15-5}$ and $\mathrm{B}_{13-9}$. This can be explained by the much stronger tendency of self-association of the PBA blocks with respect to the random ones [4], which is also true with respect to the gradient ones [32].

Dynamics of the self-assemblies

As mentioned in the introduction, systematic studies in the literature indicate that for the PBA-b-PAA block copolymers with the polymerization number of the PBA block, $n_{\mathrm{PBA}}>20$, their aqueous dispersions are kinetically frozen. The question to address now is whether the aqueous dispersions of our two block polymers, $\mathrm{B}_{15-5}$ and $\mathrm{B}_{13-9}$, with $n_{\mathrm{PBA}}=15$ and 13 , respectively, are "frozen" or dynamic.

First of all, several observations have indirectly revealed that the solutions of both $\mathrm{B}_{15-5}$ and $\mathrm{B}_{13-9}$ are dynamic, easy to reach their thermodynamic equilibrium. Specifically, the two block polymers can be easily dispersed from their dried state in alkaline water in the absence of a cosolvent, which is a good indication of dynamic nature $[14,26]$. The $\mathrm{CMC}$ can be easily measured, and the measured $\mathrm{CMC}$ depends on the PBA block length and follows the trend given by Eq. 4, as demonstrated above, which both support the dynamics of the two systems $[8,17,33]$. In fact, for "frozen" systems, their CMC is often experimentally inaccessible $[9,10,12]$, down to a dilution in $10^{-6}-10^{-7} \mathrm{w} / \mathrm{w}[15,34]$. In those cases, the measured CMC would be kinetically dependent ("apparent CMC") $[12,13]$. In addition, it is expected [17] that when the dimensionless parameter in Eq. $4, E_{\mathrm{a}} / k_{\mathrm{B}} T>16\left(T=25^{\circ} \mathrm{C}\right)$, extracting a unimer from a micelle back to water becomes difficult, i.e., the system is kinetically frozen. For our $\mathrm{B}_{15-5}$ and $\mathrm{B}_{13-9}$, the $E_{\mathrm{a}} / k_{\mathrm{B}} T$ values calculated from Eq. 5 are 12.0 and 10.9, respectively, much smaller than 16 , thus supporting their dynamics.

Second, it is well known $[9,13,15,35]$ that for spherical micelles, after their formation at the $\mathrm{CMC}$, as a thermodynamic process, upon increase in the unimer concentration, the number of the micelles increases but the micelle size and structure remain unchanged. To verify if $B_{15-5}$ and $B_{13-9}$ 
follow the same behavior, we have prepared their solutions at different concentrations and characterized by the polarized (VV) DLS. Figure 2a and $b$ shows the normalized autocorrelation functions for the solutions of $\mathrm{B}_{15-5}$ and $\mathrm{B}_{13-9}$. All the
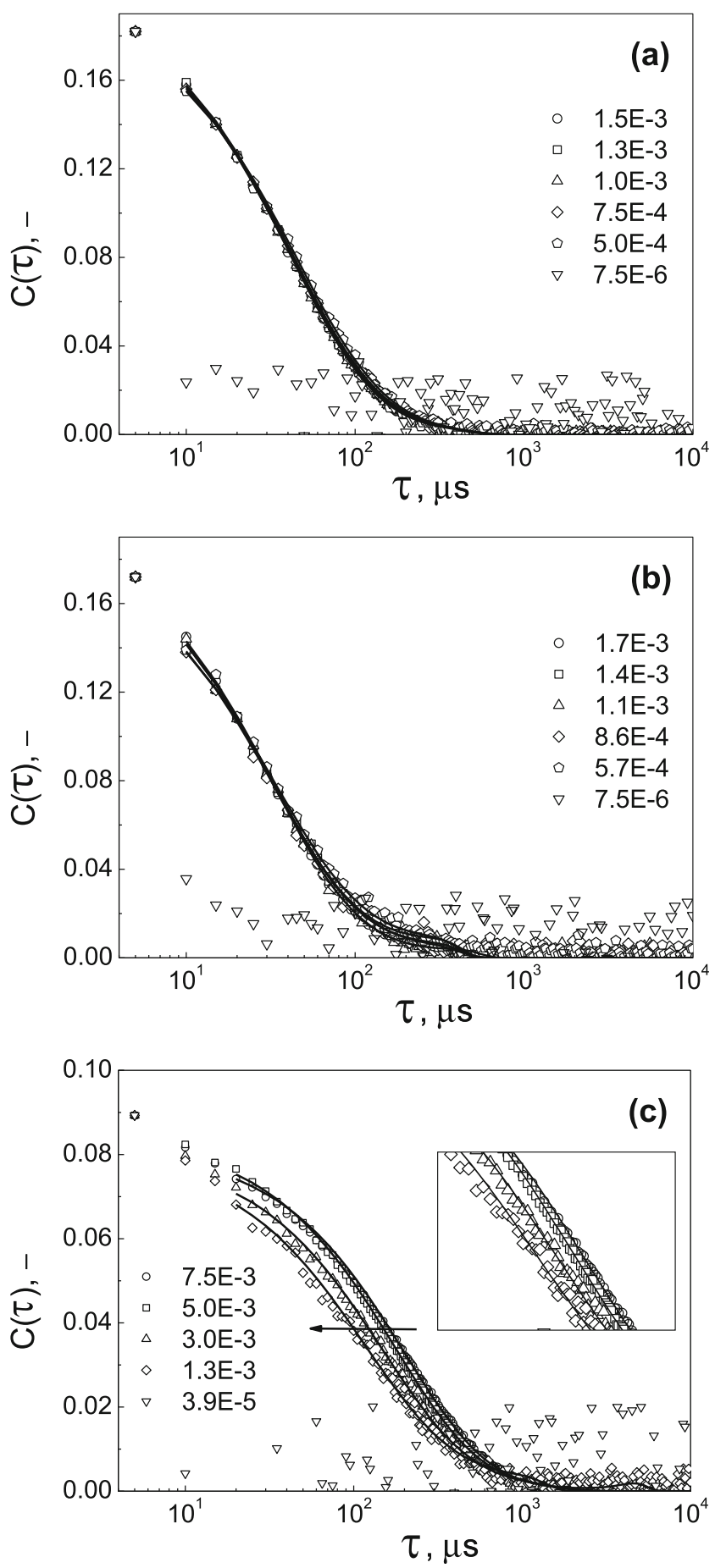

Fig. 2 Normalized autocorrelation functions from the polarized DLS for the solutions of $\mathbf{a} \mathrm{B}_{15-5}$, $\mathbf{b} \mathrm{B}_{13-9}$ and $\mathbf{c} \mathrm{R}_{13-14}$ at various concentrations (in $\mathrm{mol} / \mathrm{l}$ ) above the $\mathrm{CMC}$ (empty circle, empty square, empty triangle, empty diamond and empty pentagon) and below the CMC (empty inverted triangle). The continuous lines represent cumulant fittings of the experimental points autocorrelation functions measured at the polymer concentrations above the $\mathrm{CMC}$ have completely overlapped, and all the values of the corresponding hydrodynamic radius, $R_{\mathrm{h}}$, are within $10.0 \pm 0.06$ and $8.0 \pm 0.06 \mathrm{~nm}$, respectively, for $\mathrm{B}_{15-5}$ and $\mathrm{B}_{13-9}$. Thus, both the systems behave the same as the dynamic systems. In fact, it was observed [13] that, contrary to this behaviour, the micelles of considerably longer PBA-bPAA block copolymers exhibit concentration-dependent sizes and very low apparent $\mathrm{CMCs}\left(\sim 10^{-8} \mathrm{~mol} / \mathrm{l}\right)$.

Moreover, we have diluted the solutions of $\mathrm{B}_{15-5}$ and $\mathrm{B}_{13-9}$ from a concentration above the $\mathrm{CMC}$ to a concentration below the $\mathrm{CMC}$, and measured right afterwards the normalized autocorrelation functions, which reduce to a relatively scattered baseline, as shown in Fig. 2a and b. This clearly indicates that no micelles can be detected by the DLS. Note that the dilutions for the polymer concentrations below the CMC for the DLS measurements were selected based on the surface tension curves in Fig. 1, so as to avoid the intermediate transition regions but still with good scattering signal for the DLS measurements. This result is a further proof that aqueous dispersions of both $\mathrm{B}_{15-5}$ and $\mathrm{B}_{13-9}$ are dynamic, at their thermodynamic equilibrium.

Conversely, for the random copolymer, $\mathrm{R}_{13-14}$, the measured autocorrelation functions at different concentrations above the CMC shown in Fig. 2c exhibit a systematic shift to a smaller delay time as the polymer concentration decreases. Hence, the aggregate size decreases as the $\mathrm{R}_{13-14}$ concentration decreases (actually with the $R_{\mathrm{h}}$ value dropping from $39.2 \mathrm{~nm}$ to $24.9 \mathrm{~nm}$ along the investigated concentration range). Such behavior has also been observed for simple polyelectrolytes [4], associative polyelectrolytes [36], gradient copolymers [32], and diblock copolymers with statistical hydrophobic block [12]. When the polymer concentration is reduced below a certain value, e.g., equal to $3.9 \times 10^{-5} \mathrm{~mol} / \mathrm{l}$ in Fig. $2 \mathrm{c}$, the aggregates disappear. Therefore, the $\mathrm{CMC}$ assigned to the $\mathrm{R}_{13-14}$ aggregates is not a thermodynamic quantity but rather should be termed "apparent CMC" [12, 32].

\section{Characterization of the micelles}

In the following, we discuss the morphology only for the micelles formed by the two block polymers, $\mathrm{B}_{15-5}$ and $\mathrm{B}_{13-9}$. For the random polymer, $\mathrm{R}_{13-14}$, since the size of the aggregates ("micelles") is concentration-dependent, it is difficult to reach some concrete conclusions about their morphology, and no discussion is supplied.

\section{The morphology}

The cryo-TEM pictures of the micelles formed by $\mathrm{B}_{15-5}$ and $B_{13-9}$ are shown in Fig. 3. Due to the relatively small sizes of the objects, the resolution of the pictures is not excellent. However, two features of the micelles can still be observed: 

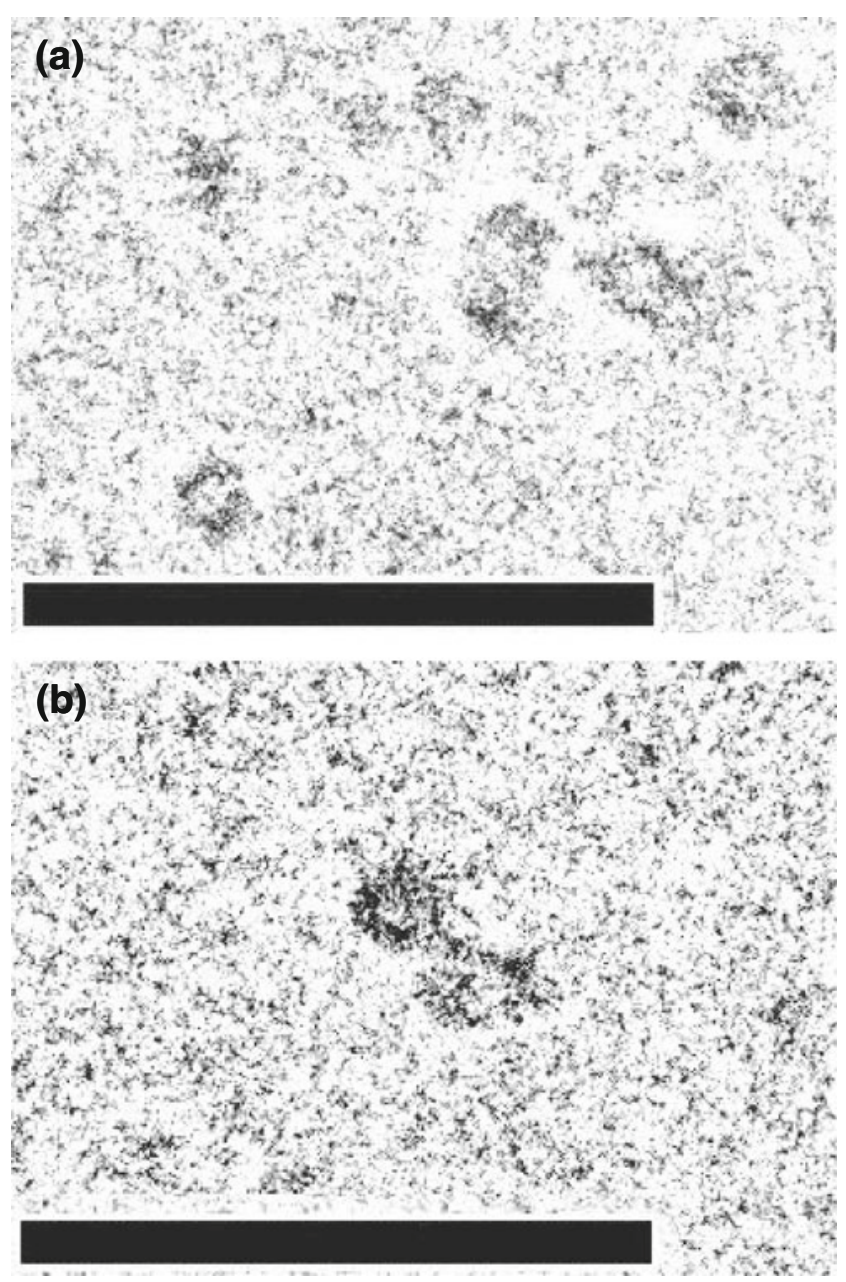

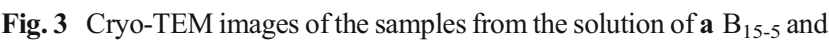
b $\mathrm{B}_{13-9}$. The scale bar corresponds to $100 \mathrm{~nm}$

(1) the micelles of both $\mathrm{B}_{15-5}$ and $\mathrm{B}_{13-9}$ are spherical, with a radius around $10 \mathrm{~nm}$, which is consistent with the hydrodynamic radius obtained above; (2) in each micelle, there is a clear darker ring that surrounds a brighter core. The latter may indicate that the micelles formed by $\mathrm{B}_{15-5}$ and $\mathrm{B}_{13-9}$ are hollow spheres (vesicles).

Let us first verify the spherical geometry of the micelles. Toward this aim, we have performed depolarized (VH) DLS for the solutions of $\mathrm{B}_{15-5}$ and $\mathrm{B}_{13-9}$ at a concentration above the CMC. It turns out that, unlike the cases of the polarized (VV) DLS in Fig. 2, no autocorrelation functions can be determined, and in fact, the intensity becomes negligible during the depolarized DLS measurements performed at different angles. Thus, no decay rates can be defined from the depolarized DLS measurements. This indicates that the micelles are optically isotropic [37], confirming their spherical geometry.

To confirm the vesicle structure, we recall the measured hydrodynamic radii of the micelles, which are $R_{\mathrm{h}}=10.0$ and $8.0 \mathrm{~nm}$, respectively, for $\mathrm{B}_{15-5}$ and $\mathrm{B}_{13-9}$, as listed in Table 2 . Then, if the micelles are homogeneous spheres, the length of a
Table 2 Characteristics of the micelles formed by two block copolymers

\begin{tabular}{llllll}
\hline Surfactant & $M_{\mathrm{w}, \mathrm{m}}\left(\mathrm{g} \mathrm{mol}^{-1}\right)$ & $N_{\mathrm{agg}, \mathrm{m}}$ & $R_{\mathrm{g}}(\mathrm{nm})$ & $R_{\mathrm{h}}(\mathrm{nm})$ & $R_{\mathrm{g}} / R_{\mathrm{h}}$ \\
\hline $\mathrm{B}_{15-5}$ & $4.22 \times 10^{5}$ & 134 & 10.3 & 10.0 & 1.03 \\
$\mathrm{~B}_{13-9}$ & $1.80 \times 10^{5}$ & 55 & 11.7 & 8.0 & 1.46
\end{tabular}

unimer should be around $10.0 \mathrm{~nm}$ and $8.0 \mathrm{~nm}$. Let us assume that both the PBA and PAA blocks in each unimer are in their fully stretched configuration. Considering the length of each monomer unit being $0.25 \mathrm{~nm}[13,38]$, we have the maximum length of $5 \mathrm{~nm}$ for a $B_{15-5}$ unimer and $5.5 \mathrm{~nm}$ for a $B_{13-9}$ unimer, which both are much smaller than the corresponding $R_{h}$ value, thus confirming the vesicle structure of the micelles. This result is consistent with the literature observations that for ionic diblock micelles with the hydrophobic block longer than the hydrophilic one, morphologies like cylinders, vesicles and lamellae are favored [2, 5-10]. Therefore, a schematic illustration of our vesicles is given in Fig. 4, where the hydrophobic blocks of the two layers in the middle form the hydrophobic core, while the hydrophilic blocks of each layer form the inner and outer coronae.

\section{The aggregation number}

The classical SLS (Zimm plot) experiments, as described in section "Static and dynamic light scattering", have been carried out for the solutions of $\mathrm{B}_{15-5}$ and $\mathrm{B}_{13-9}$ at concentrations above the CMC. The results are shown in Fig. 5, from which the molar mass and radius of gyration, $M_{\mathrm{w}, \mathrm{m}}$ and $R_{\mathrm{g}}$, of the micelles can be determined, which are collected in Table 2 . Then, the corresponding aggregation number, $N_{\mathrm{agg}, \mathrm{m}}$, can be computed from the molecular weight of a unimer, $M_{\mathrm{w}}[36]$ :

$N_{\mathrm{agg}, \mathrm{m}}=\frac{M_{\mathrm{w}, \mathrm{m}}}{M_{\mathrm{w}}}=\frac{M_{\mathrm{w}, \mathrm{m}}}{\mathrm{PDI} \cdot M_{\mathrm{n}}}$,

With the values of the parameters in Tables 1 and 2, the obtained values of $N_{\mathrm{agg}, \mathrm{m}}$ are 134 and 55, respectively, for the micelles of $\mathrm{B}_{15-5}$ and $\mathrm{B}_{13-9}$. The $N_{\mathrm{agg}, \mathrm{m}}$ value of $\mathrm{B}_{15-5}$ is more than twice that of $\mathrm{B}_{13-9}$. This agrees with the general trend that $N_{\text {agg,m }}$ decreases as the hydrophobic block length decreases $[8,10,26,33,39]$ and as the hydrophilic block length increases $[10,14,39]$. The latter dependence is strong for crewcut micelles [40] or generally when the hydrophobic block is short enough [27,33].

The $R_{\mathrm{g}}$ value of the micelles estimated from the Zimm plot is rather comparable to the corresponding $R_{\mathrm{h}}$ value, with a $R_{\mathrm{g}} /$ $R_{\mathrm{h}}$ value very close to 1 in Table 2 . This further confirms the hollow sphere morphology of the micelles [10, 41-43]. Note that for $\mathrm{B}_{13-9}$ the $R_{\mathrm{g}} / R_{\mathrm{h}}$ value is significantly larger than 1 . This is presumably due to polydispersity, which is known to increase $R_{\mathrm{g}} / R_{\mathrm{h}}[39,41,43]$. 
Fig. 4 Schematic illustration of a vesicle formed by the block copolymer $\mathrm{B}_{15-5}$ or $\mathrm{B}_{13-9}$

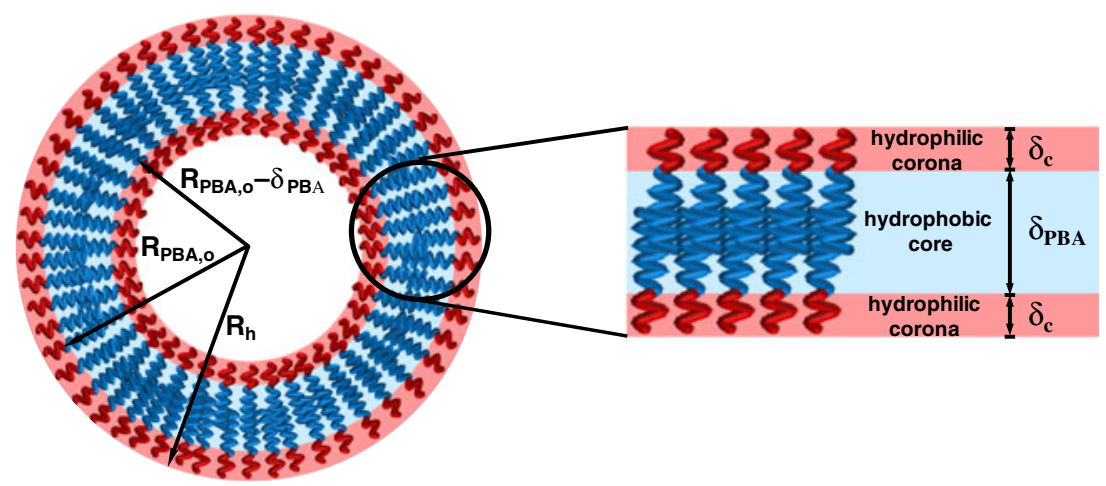

Thickness of each layer in the vesicle

The $\mathrm{pH}$ value of all our experiments is 7.0 , at which the dissociation degree of the PAA chains should be high, 85$90 \%$ [13]. Then, in the absence of additional electrolytes, the PAA chains can be considered to be fully stretched $[33,38$, 44]. Thus, the thickness of the inner and outer coronae, $\delta_{\mathrm{c}}$, can be estimated to be $0.25 \times n_{\mathrm{PAA}}$, where $n_{\mathrm{PAA}}$ is the polymerization number of the PAA block, leading to $\delta_{\mathrm{c}}=1.25$ and $2.25 \mathrm{~nm}$, respectively, for the coronae of $\mathrm{B}_{15-5}$ and $\mathrm{B}_{13-9}$.

To evaluate the thickness of the PBA core, $\delta_{\mathrm{PBA}}$, we assume that the density of the PBA core equals that of bulk $\operatorname{PBA}\left(\rho_{\mathrm{PBA}}=1.10 \mathrm{~g} \mathrm{~cm}^{-3}\right.$ at $\left.T=25^{\circ} \mathrm{C}[28]\right)$. With $\rho_{\mathrm{PBA}}$ and the aggregation number, $N_{\text {agg,m }}$, we can compute the volume of the PBA core, $V_{\mathrm{PBA}}=388.4$ and $138 \mathrm{~nm}^{3}$, respectively, for the case of $\mathrm{B}_{15-5}$ and $\mathrm{B}_{13-9}$. On the other hand, we can consider that the outer radius of the PBA core equals the hydrodynamic radius of the micelles minus the thickness of the outer PAA corona, i.e., $R_{\mathrm{PBA}, \mathrm{o}}=R_{\mathrm{h}}-\delta_{\mathrm{c}}=8.75$ and $5.75 \mathrm{~nm}$, for $\mathrm{B}_{15-5}$ and $\mathrm{B}_{13-9}$. Therefore, from

$V_{\mathrm{PBA}}=\frac{4}{3} \cdot \pi \cdot\left[R_{\mathrm{PBA}, \mathrm{o}}^{3}-\left(R_{\mathrm{PBA}, \mathrm{o}}-\delta_{\mathrm{PBA}}\right)^{3}\right]$,

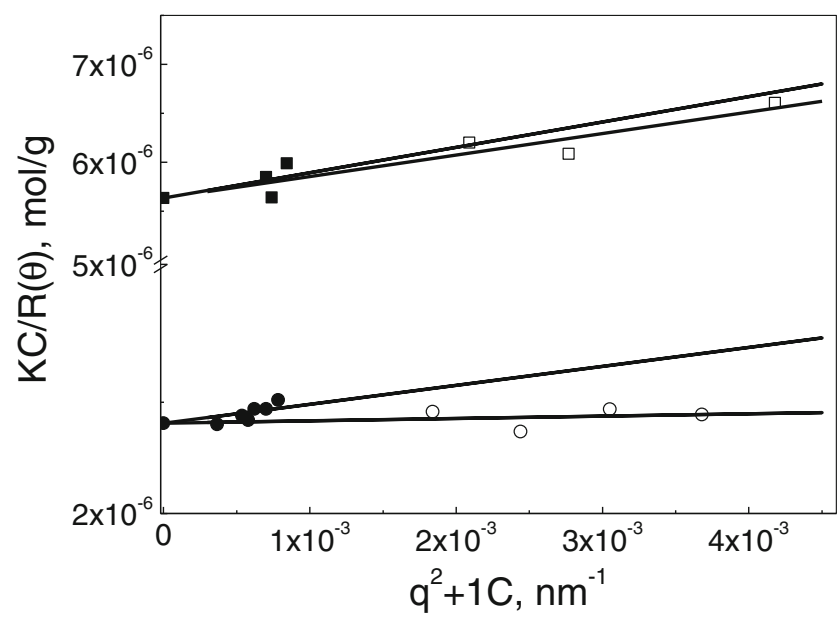

Fig. 5 Zimm plots for block copolymers, B15-5 (circles) and B13-9 (squares). Open symbols correspond to extrapolation to zero angle, while filled symbols to zero concentration we obtain $\delta_{\mathrm{PBA}}=0.42$ and $0.35 \mathrm{~nm}$, respectively, for $\mathrm{B}_{15-5}$ and $\mathrm{B}_{13-9}$. Let us check if the estimated $\delta_{\mathrm{PBA}}$ values are meaningful, by assuming that the PBA block in its collapsed state attains $18 \%$ of its contour length, as estimated by Battaglia and Ryan [45] for the hydrophobic block in poly(ethylene oxide)-b-polybutadiene vesicles. Without assuming any overlapping between the two opposite layers in the hydrophobic core, this leads to $\delta_{\mathrm{PBA}}=1.35$ and $1.17 \mathrm{~nm}$, respectively, for the case of $\mathrm{B}_{15-5}$ and $\mathrm{B}_{13-9}$, which, though somewhat larger, are rather comparable to our above estimates. Of course, if one assumes full overlapping between the two PBA layers, the agreement should be considered to be excellent.

\section{Concluding remarks}

Two short block copolymers, PBA-b-PAA (poly-butyl acrylate-b-poly-acrylic acid), have been synthesized with the polymerization numbers of the PBA and PAA blocks, 15 and 5, and 13 and 9, thus, referred to as $\mathrm{B}_{15-5}$ and $\mathrm{B}_{13-9}$, respectively. Their self-association dynamics and morphology in water have been studied by combining SLS (Zimm plot), polarized and depolarized DLS, and cryo-TEM experiments. Their behaviors have been compared with those of a random PBA-co-PAA copolymer composed of 13 BA and 14 AA units, referred to as $\mathrm{R}_{13-14}$.

Evidences and data have confirmed that the selfassociation process of both $\mathrm{B}_{15-5}$ and $\mathrm{B}_{13-9}$ in water is dynamic, instead of frozen as observed in the literature for the same block copolymers but with larger polymerization numbers of the PBA block (>20). These evidences include: (1) their dried state can be easily dissolved in water and reach equilibrium; (2) the measured CMC agrees with the theoretical prediction, not only in the orders of magnitude and trend (with respect to sizes of PBA and PAA), but, in the case of $\mathrm{B}_{13-9}$, also in the absolute value; (3) the size of the formed micelles is independent of the polymer concentration; (4) the formed micelles disappear easily when their solution is progressively diluted below the CMC. 
The cryo-TEM images indicate that the formed micelles are hollow spheres (vesicles). The spherical shape has been confirmed by the depolarized DLS. The values of the hydrodynamic radius $\left(R_{\mathrm{h}}\right)$ measured by the polarized DLS (10.0 and $8.0 \mathrm{~nm}$, respectively, for $\mathrm{B}_{15-15}$ and $\mathrm{B}_{13-9}$ ) support the hollow morphology of the micelles, because the evaluated length of individual molecules of the two polymers, even at their fully stretched state, is only around $1 / 2 R_{\mathrm{h}}$, thus unable to form a homogeneous sphere of the given size. Furthermore, the ratio, $R_{\mathrm{h}} / R_{\mathrm{g}}$ (where $R_{\mathrm{g}}$ is the radius of gyration obtained from SLS), being close to 1 , also supports the hollow sphere morphology. In addition, the aggregation number of the micelles has been determined from the Zimm plot, which allows us to estimate the thickness of the hydrophobic PBA layer.

Regarding the random copolymer, $\mathrm{R}_{13-14}$, they also selfassemble into micelle-like aggregates, with a CMC roughly one order of magnitude larger than those of the two block copolymers. However, their size appears to monotonically decrease with decreasing concentration. Therefore, the respective $\mathrm{CMC}$ should be a non-equilibrium, apparent $\mathrm{CMC}$, despite the fact that the aggregates also disappear when the concentration is below it.

Acknowledgments Financial support from Swiss National Science Foundation (Grant No. 200020_147137/1) and China Scholarship Council is gratefully acknowledged. We thank Dr. T. Ishikawa for performing cryo-TEM image and EMEZ of ETH Zurich for allocating beam time, as well as Professor M. Morbidelli and M. Lattuada for useful discussion.

\section{References}

1. Alexandridis P, Lindman B (eds) (2000) Amphiphilic block copolymers: self-assembly and applications. Elsevier, Amsterdam

2. Hamley IW (1998) The physics of block copolymers. Oxford Univ. Press, New York

3. Xie DL, Lamprou A, Storti G, Morbidelli M, Wu H (2012) Shearinduced gelation of soft strawberry-like particles in the presence of polymeric P(BA-b-AA) surfactants. Phys Chem Chem Phys 14(41): 14374-14382. doi:10.1039/C2CP42417G

4. Fleer GJ, Cohen Stuart MA, Scheutjens JMHM, Cosgrove T, Vincent B (1993) Polymers at interfaces. Chapman \& Hall, London

5. Zhang LF, Eisenberg A (1995) Multiple morphologies of crew-cut aggregates of polystyrene-b-poly(acrylic acid) block-copolymers. Science 268(5218):1728-1731. doi:10.1126/science.268.5218.1728

6. Fu G-D, Li GL, Neoh KG, Kang ET (2011) Hollow polymeric nanostructures - synthesis, morphology and function. Prog Polym Sci 36(1):127-167. doi:10.1016/j.progpolymsci.2010.07.011

7. Meng F, Zhong Z, Feijen J (2009) Stimuli-responsive polymersomes for programmed drug delivery. Biomacromolecules 10(2):197-209. doi:10.1021/bm801127d

8. Pioge S, Fontaine L, Gaillard C, Nicol E, Pascual S (2009) Selfassembling properties of well-defined poly(ethylene oxide)-b-poly (ethyl acrylate) diblock copolymers. Macromolecules 42(12):42624272. doi: $10.1021 / \mathrm{ma} 802705 \mathrm{~b}$

9. Borisov O, Zhulina E, Leermakers F, Müller A (2011) Selfassembled structures of amphiphilic ionic block copolymers: theory, self-consistent field modeling and experiment. In: Müller AHE,
Borisov O (eds) Advances in polymer science, vol 241. Springer, Berlin, pp 57-129. doi:10.1007/12 2011 114

10. Forster S, Zisenis M, Wenz E, Antonietti M (1996) Micellization of strongly segregated block copolymers. J Chem Phys 104(24):99569970. doi:10.1063/1.471723

11. Nicolai T, Colombani O, Chassenieux C (2010) Dynamic polymeric micelles versus frozen nanoparticles formed by block copolymers. Soft Matter 6(14):3111-3118. doi:10.1039/B925666K

12. Bendejacq DD, Ponsinet V, Joanicot M (2005) Chemically tuned amphiphilic diblock copolymers dispersed in water: from colloids to soluble macromolecules. Langmuir 21(5):1712-1718. doi:10. 1021/la048983r

13. Colombani O, Ruppel M, Schubert F, Zettl H, Pergushov DV, Muller AHE (2007) Synthesis of poly(n-butyl acrylate)-block-poly(acrylic acid) diblock copolymers by ATRP and their micellization in water. Macromolecules 40(12):4338-4350. doi:10.1021/ma0609578

14. Colombani O, Ruppel M, Burkhardt M, Drechsler M, Schumacher M, Gradzielski M, Schweins R, Muller AHE (2007) Structure of micelles of poly(n-butyl acrylate)-block-poly(acrylic acid) diblock copolymers in aqueous solution. Macromolecules 40(12):43514362. doi: $10.1021 / \mathrm{ma} 0609580$

15. Jacquin M, Muller P, Talingting-Pabalan R, Cottet H, Berret JF, Futterer T, Theodoly O (2007) Chemical analysis and aqueous solution properties of charged amphiphilic block copolymers PBA-bPAA synthesized by MADIX((c)). J Colloid Interface Sci 316:897911. doi:10.1016/j.jcis.2007.08.025

16. Theodoly O, Jacquin M, Muller P, Chhun S (2009) Adsorption kinetics of amphiphilic diblock copolymers: from kinetically frozen colloids to macrosurfactants. Langmuir 25(2):781-793. doi:10.1021/ la8030254

17. Jacquin M, Muller P, Cottet H, Theodoly O (2010) Self-assembly of charged amphiphilic diblock copolymers with insoluble blocks of decreasing hydrophobicity: from kinetically frozen colloids to macrosurfactants. Langmuir 26(24):18681-18693. doi:10.1021/ la103391p

18. Lejeune E, Drechsler M, Jestin J, Muller AHE, Chassenieux C, Colombani O (2010) Amphiphilic diblock copolymers with a moderately hydrophobic block: toward dynamic micelles. Macromolecules 43(6):2667-2671. doi:10.1021/ma902822g

19. Lejeune E, Chassenieux C, Colombani O (2011) pH induced desaggregation of highly hydrophilic amphiphilic diblock copolymers. In: Starov VPK (ed) Trends in colloid and interface science XXIV, vol 138, Progress in Colloid and Polymer Science., pp 7-16. doi:10.1007/978-3-642-19038-4 2

20. Halperin A, Alexander S (1989) Polymeric micelles — their relaxation kinetics. Macromolecules 22(5):2403-2412. doi:10.1021/ ma00195a069

21. Charbonneau C, Chassenieux C, Colombani O, Nicolai T (2011) Controlling the dynamics of self-assembled triblock copolymer networks via the $\mathrm{pH}$. Macromolecules 44(11):4487-4495. doi:10.1021/ ma2002382

22. Dimitrov I, Trzebicka B, Muller AHE, Dworak A, Tsvetanov CB (2007) Thermosensitive water-soluble copolymers with doubly responsive reversibly interacting entities. Prog Polym Sci 32(11):12751343. doi:10.1016/j.progpolymsci.2007.07.001

23. Haney M (2011) Determination of $\mathrm{dn} / \mathrm{dc}$ by multi-point RI method, Viscotek GPC Application Notes. Viscotek Corporation, Houston

24. $\mathrm{Wu} \mathrm{H}$ (2010) Correlations between the Rayleigh ratio and the wavelength for toluene and benzene. Chem Phys 367(1):44-47. doi:10. 1016/j.chemphys.2009.10.019

25. Namani T, Ishikawa T, Morigaki K, Walde P (2007) Vesicles from docosahexaenoic acid. Colloids and Surf B-Biointerfaces 54(1):118 123. doi:10.1016/j.colsurfb.2006.05.022

26. Gaillard N, Guyot A, Claverie J (2003) Block copolymers of acrylic acid and butyl acrylate prepared by reversible addition-fragmentation chain transfer polymerization: synthesis, characterization, and use in 
emulsion polymerization. J Polym Sci Part a-Polymer Chem 41(5): 684-698. doi:10.1002/pola.10606

27. Astafieva I, Khougaz K, Eisenberg A (1995) Micellization in block polyelectrolyte solutions: 2 . Fluorescence study of the critical micelle concentration as a function of soluble block length and salt concentration. Macromolecules 28(21):7127-7134. doi:10.1021/ ma00125a015

28. Barudio I, Févotte G, McKenna TF (1999) Density data for copolymer systems: butyl acrylate/vinyl acetate homo- and copolymerization in ethyl acetate. Eur Polym J 35(5):775-780. doi:10.1016/ S0014-3057(98)00070-6

29. Lee DY, Kim JH (1998) Preparation of small-sized carboxylated latexes by emulsion polymerization using alkali-soluble random copolymer. J Appl Polym Sci 69(3):543-550. doi:10.1002/(SICI) 1097-4628(19980718)

30. Hwu HD, Lee YD (2000) Studies of alkali soluble resin as a surfactant in emulsion polymerization. Polymer 41(15):5695-5705. doi:10. 1016/S0032-3861(99)00762-4

31. Kuo PL, Chen CJ (1993) Functional polymers for colloidal applications: 5. Novel behavior of polymeric emulsifiers in emulsion polymerization. J Polym Sci Part a-Polym Chem 31(1):99-111. doi:10. 1002/pola.1993.080310112

32. Lefay C, Charleux B, Save M, Chassenieux C, Guerret O, Magnet S (2006) Amphiphilic gradient poly(styrene-co-acrylic acid) copolymer prepared via nitroxide-mediated solution polymerization. Synthesis, characterization in aqueous solution and evaluation as emulsion polymerization stabilizer. Polymer 47(6):1935-1945. doi: 10.1016/j.polymer.2006.01.034

33. Burguiere C, Chassenieux C, Charleux B (2003) Characterization of aqueous micellar solutions of amphiphilic block copolymers of poly(acrylic acid) and polystyrene prepared via ATRP. Toward the control of the number of particles in emulsion polymerization. Polymer 44(3):509-518. doi:10.1016/S0032-3861(02)00811-X

34. Rager T, Meyer WH, Wegner G, Winnik MA (1997) Influence of chain length and salt concentration on block copolymer micellization. Macromolecules 30(17):4911-4919. doi:10.1021/ma9618661

35. Kahlweit M, Teubner M (1980) On the kinetics of micellization in aqueous-solutions. Adv Colloid Interf Sci 13(1-2):1-64. doi:10. 1016/0001-8686(80)87001-1

36. Sedlak M (1999) What can be seen by static and dynamic light scattering in polyelectrolyte solutions and mixtures? Langmuir 15(12):4045-4051. doi:10.1021/la981189j

37. Zimbone M, Calcagno L, Messina G, Baeri P, Compagnini G (2011) Dynamic light scattering and UV-vis spectroscopy of gold nanoparticles solution. Mater Lett 65(19-20):2906-2909. doi:10.1016/j. matlet.2011.06.054

38. Eghbali E, Colombani O, Drechsler M, Muller AHE, Hoffmann H (2006) Rheology and phase behavior of poly(n-butyl acrylate)-blockpoly(acrylic acid) in aqueous solution. Langmuir 22(10):4766-4776. doi:10.1021/la053272u

39. Qin AW, Tian MM, Ramireddy C, Webber SE, Munk P, Tuzar Z (1994) Polystyrene poly(methacrylic acid) block-copolymer micelles. Macromolecules 27(1):120-126. doi:10.1021/ma00079a018

40. Borisov OV, Zhulina EB (2002) Effect of salt on self-assembly in charged block copolymer micelles. Macromolecules 35(11):44724480. doi:10.1021/ma010934n

41. Mossmer S, Spatz JP, Moller M, Aberle T, Schmidt J, Burchard W (2000) Solution behavior of poly(styrene)-block-poly(2-vinylpyridine micelles containing gold nanoparticles. Macromolecules 33(13):4791-4798. doi:10.1021/ma992006i

42. Stauch O, Schubert R, Savin G, Burchard W (2002) Structure of artificial cytoskeleton containing liposomes in aqueous solution studied by static and dynamic light scattering. Biomacromolecules 3(3): 565-578. doi:10.1021/bm0200074

43. Burchard W (1983) Static and dynamic light scattering from branched polymers and biopolymers. In: Light dcattering from polymers, vol 48. Advances in Polymer Science. Springer, Berlin, pp 1124. doi:10.1007/3-540-12030-0 1

44. van der Maarel JRC, Groenewegen W, Egelhaaf SU, Laap A (2000) Salt-induced contraction of polyelectrolyte diblock copolymer micelles. Langmuir 16(19):7510-7519. doi:10.1021/la000299z

45. Battaglia G, Ryan AJ (2005) Bilayers and interdigitation in block copolymer vesicles. J Am Chem Soc 127(24):8757-8764. doi:10. $1021 / \mathrm{ja} 050742 \mathrm{y}$ 